
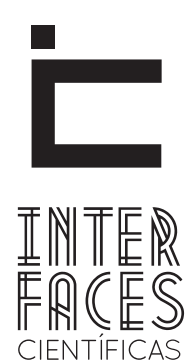

SAÚDE E AMBIENTE

ISSN IMPRESSO 2316-3313

E - ISSN 2316-3798

DOI - 10.17564/2316-3798.2017v5n2p33-40

\title{
FIBROMA OSSIFICANTE CENTRAL EM MANDÍBULA: RELATO DE CASO
}

\author{
CENTRAL OSSIFYING FIBROMA IN THE MANDIBLE: A CASE REPORT \\ FIBROMA OSIFICANTE CENTRAL EN LA MANDÍBULA: REPORTE DE UN CASO
}

\author{
Francisco de Assis Almeida Lima Júnior ${ }^{1}$ \\ Candice Rebouças Rosa ${ }^{3}$ \\ Maria de Fátima Batista de Melo $^{5}$
}

\author{
John Lennon Silva Cunha ${ }^{2}$ \\ Juliana Batista Melo da Fonte ${ }^{4}$ \\ Ricardo Luiz Cavalcanti de Albuquerque Júnior ${ }^{6}$
}

\section{RESUMO}

O Fibroma Ossificante Central (FOC) é uma neoplasia benigna de origem fibro-óssea incomum que acomete os ossos da face, caracterizado pela substituição da arquitetura óssea normal por tecido conjuntivo rico em fibroblastos e fibras colágenas, com deposição de material mineralizado em quantidade e morfologia variada. Estudos tem demonstrado a existência de muita superposição entre os aspectos clínicos, imaginológicos e histopatológicos das lesões fibro-ósseas benignas dos maxilares, determinando dificuldades no estabelecimento de um diagnóstico seguro. 0 objetivo deste trabalho foi relatar um caso de FOC em uma localização incomum, com ênfase na discussão de sua etiopatogênese e critérios de diagnóstico di- ferencial imaginológico e histopatológico entre esta entidade e a outras lesões fibro-ósseas dos maxilares, como a displasia óssea fibrosa e o fibroma ossificante juvenil agressivo. A análise do caso e sua relação com a literatura vigente permitiu concluir que é de extrema relevância que o diagnóstico do FOC seja devidamente embasado numa correlação de achados clinicopatológicos para assegurar o estabelecimento do diagnóstico e terapêutica mais apropriados.

\section{PALAVRAS-CHAVES}

Lesões Fibro-ósseas Benigna. Fibroma Ossificante Central. Diagnóstico Diferencial. 


\section{ABSTRACT}

Central ossifying fibroma (COF) is an uncommon benign tumor of fibro-osseous origin that affects the jaws. It is characterized by the replacement of the conventional bone architecture for a fibroblast-rich connective tissue and collagen fibers, associated with formation of calcified material of varied amount and morphology. It has been reported a considerable overlapping of the clinic, imaginologic and histopathologic features of fibro-osseous lesions of the jaws, which has provided difficulties in the establishment of a conclusive diagnosis. The purpose of this paper was to report a case of COF in an unusual anatomic location, with highlights in the discussion of the etiopathogenesis and parameters of imaginologic and histopathologic differential diagnosis between this tumor and other fibro-osseous lesions, such as fibrous dysplasia and juvenile ossifying fibroma. The analysis of the case, as well as its relationship with the current specialized literature leads to the conclusion that the correlation of clinicopathologic findings is extremely important to assure the establishment of the correct diagnosis and therapeutic approach in cases of COF.

\section{KEYWORDS}

Benign fibro-osseous lesions. Central ossifying fibroma. Differential diagnosis.

\section{RESUMEN}

El fibroma osificante central (FOC) es un tumor benigno de origen óseo fibroso poco frecuente que afecta a los huesos de la cara, que se caracteriza por la sustitución de la arquitectura normal de la médula por tejido conectivo rico en fibroblastos y fibras de colágeno, con depósito de material mineralizado en cantidad y morfología variada. Los estudios muestran que hay mucha superposición entre los aspectos clínicos, de imágenes y lahistopatología de las lesiones fibro-óseas benignas de la mandíbula que conllevan a dificultades para establecer un diagnóstico seguro. El objetivo de este estudio fue relatar un caso de FOC en una localización poco común, haciendo hincapié en la discusión de su etiopatogenia y en los criterios dife- renciales de imagen e histopatologia de esta entidad con otras lesiones fibro-óseas de los maxilares como la displasia ósea fibrosa y el fibroma osificante agresivo juvenil. El análisis del caso y su relación con la literatura actual permitieron concluir que es de suma importancia que el diagnóstico de FOC sea realizado correlacionando los hallazgos clínicos patológicos para garantizar el establecimiento del diagnóstico y el tratamiento más adecuado.

\section{PALABRAS CLAVE}

lesiones fibro-óseas benignas; fibroma osificante central; diagnóstico diferencial. 


\section{INTRODUÇ̃̃̃o}

O Fibroma Ossificante Central (FOC) é uma neoplasia benigna de origem fibro-óssea incomum que acomete os ossos da face, descrita pela primeira vez por Menzel em 1842 (HUNASGI; RAGHUNA$\mathrm{TH}$, 2012). É caracterizado pela substituição da arquitetura óssea normal por tecido conjuntivo rico em fibroblastos e fibras colágenas, com deposição de material mineralizado em quantidade e morfologia variada (KONOPKA ET AL., 2012).

Clinicamente, o FOC apresenta-se de forma assintomática, predileção por indivíduos do sexo feminino, com relação aproximada de 4:1, ocorrendo especialmente entre a $3^{a}$ e $4^{a}$ década de vida, sendo a região posterior de mandíbula o sítio anatômico mais acometido (TRIANTAFILLIDOU ET AL., 2012). Apesar de usualmente apresentar desenvolvimento lento, esta lesão pode manifestar-se em alguns casos de forma agressiva, com alto grau de recidiva, provocando deformidade moderada a severa (VIEIRA-QUIEROZ ET AL., 2013). Apesar de raros, existem relatos de FOCs bilaterais (RIBEIRO ET AL., 2011), bimaxilares (DESAI ET AL., 2013) ou múltiplos (WANG ET AL., 2014). Além disso, a associação sincrônica entre FOC e outras lesões tem sido descritas, como a Lesão Central de Células Gigantes (FASOLIS ET AL., 2013) e o Fibroma Odontogênico Central (SILVA ET AL., 2007).

Os achados imaginológicos do FOC dependem do seu estágio de desenvolvimento, mas a maioria dos casos se manifesta como uma área de osteólise circunscrita com delimitação nítida e esclerótica em relação ao osso adjacente, podendo ser uni ou multiloculares. Quando estão no período maduro, podem consistir em uma massa radiopaca densa, também circunscrita e de delimitação radiotransparente (PHATTARATARATIP; PHOLJAROEN; TIRANON, 2013).

Histopatologicamente, os FOCs apresentam-se como lesões delimitadas por corticais ósseas não curvilíneas, imersas ao um tecido conjuntivo fibroso, geralmente com padrão não lamelar (HUNASGI; RAGHUNATH, 2012). Além disso, há presença de depósitos mineralizados esferoidais semelhantes ao cemento, contribuindo para a maior variabilidade fenotípica do material mineralizado depositado nestas lesões (KONOPKA ET AL., 2012). Segundo Oliveira e outros autores (2008), a presença de pavimentação osteoblástica é um achado relativamente frequente nos casos de FOCs, sendo observada em aproximadamente (46,7\%).

Por sua natureza expansiva osteodestrutiva, o tratamento de escolha para o FOC é a excisão cirúrgica seguida de curetagem da loja óssea remanescente (FASOLIS ET AL., 2013). Em caso de lesões muito extensas, tem sido preconizado o uso da técnica de Le Fort I para realização de abordagem cirúrgica. Esta técnica preconiza a realização de uma fratura induzida que se estende de forma horizontal da pirâmide nasal para os processos pterigoides, e apresenta as vantagens de propiciar uma linha de visão mais direta e melhor exposição da lesão, além de oferecer um melhor resultado estético (LAUREANO FILHO ET AL., 2004). A recorrência é considerada rara, e, quando ocorre, é mais comum em pacientes jovens (AGARWAL ET AL., 2013).

Diante do exposto, o objetivo deste trabalho foi relatar um caso de FOC em uma localização incomum, com ênfase na discussão de critérios de diagnóstico diferencial entre esta entidade e as demais lesões fibro-ósseas dos maxilares.

\section{RELATO DO CASO}

Paciente do sexo feminino, 48 anos de idade, residente na cidade de Aracaju-SE, compareceu a consultório odontológico privado, com queixa de um aumento de volume na região mentoniana, assintomática, com evolução de mais de um ano.

Ao exame físico intraoral foi observado aumento da cortical óssea lingual na região de sínfise mandi- 
bular, com mucosa normocorada, próximo aos dentes 31, 32 e 33, sem deslocamento dental, de consistência pétrea, com aproximadamente $2,0 \mathrm{~cm}$. Na região vestibular não foi evidenciada alteração digna de nota (FIGURA 1).

\section{1 - Aspecto clínico intraoral da paciente}

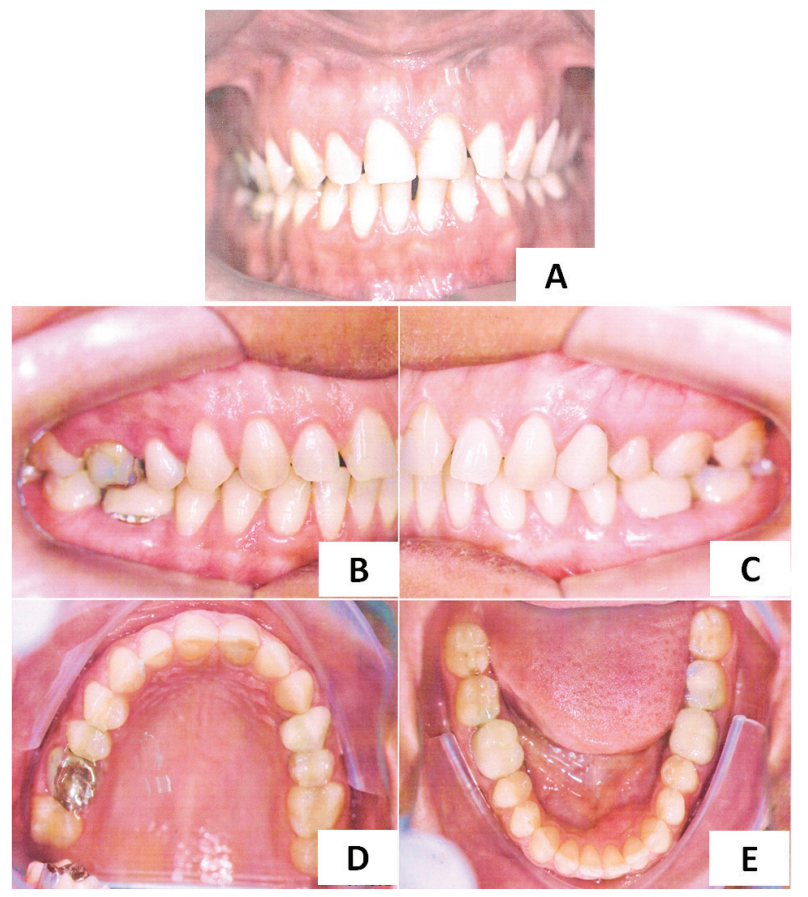

Fonte: Dados da pesquisa

Durante o exame físico extraoral foram notados linfonodos ovalados infracentimétricos submentonianos, submandibulares e júgulo-carotídeos altos sem maior significado clínico.

Foi realizada tomografia computadorizada com feixe cônico (cone beam), na qual se pôde observar uma imagem mista localizada na região mentoniana (paramediana esquerda), em contato com processos alveolares e região periapical dos dentes 31,32 e 33, com aspecto levemente insuflativo, contendo uma matriz cálcica, expressa sob a forma de massas hiperdensas, disposta em meio a massas hipoden- sas irregulares. Não foram identificados sinais imaginológicos de ruptura das corticais ósseas. A lesão apresentava dimensões de 1,8 x 1,2 x 1,4 cm (T/A/L), sugerindo uma lesão fibro-óssea benigna (FIGURA 2).

Figura 2 - Aspecto imaginológico da lesão em cortes axiais de tomografia computadorizada de feixe cônico

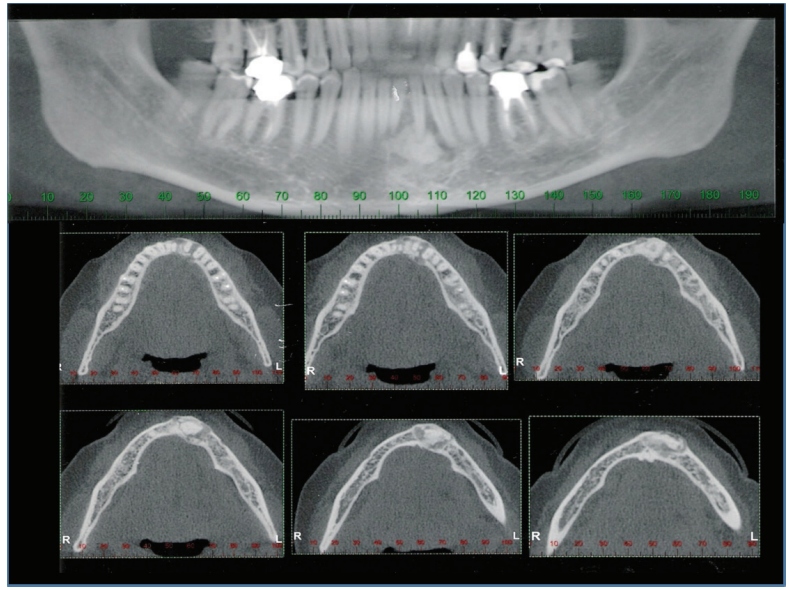

Fonte: Dados da pesquisa

Devido seu caráter benigno foi realizada então uma biopsia excisional, com remoção cirúrgica completa da lesão seguida de curetagem da loja óssea remanescente. Atualmente, a paciente encontra-se sob proservação e, após um ano de acompanhamento, não foram observados sinais de recidiva da lesão.0 espécime cirúrgico foi remetido para análise histopatológica.

O padrão histológico da lesão foi caracterizado por uma proliferação fusocelular organizada em fascículos curtos entrecruzados, imersos em um estroma fibroso de moderada densidade. De permeio, foi evidenciada a deposição de material mineralizado, ora trabecular, ora psamomatóide, com atividade osteoblástica evidente (FIGURA 3). Foi realizada também uma coloração especial em Picrossírius (luz polarizada) onde se pode verificar um estroma fibrilar birrefringente dourado, sugerindo acúmulo de colágeno fibroso tipo I e trabéculas ósseas densamente birrefringentes (birrefringência dourada) também consti- 
tuídas predominantemente de colágeno tipo I (FIGURA 3). Esses achados determinaram o diagnóstico de Fibroma Ossificante Central.

Figura 3 - Secções histológicas da lesão. (A) e (B) proliferação fusocelular fasciculada e deposição de trabéculas ósseas (HE, 100x e 400x). Secções coradas em tricrômico de Masson (C) e picrossírius (D) mostrando colagenização do estroma (400x)

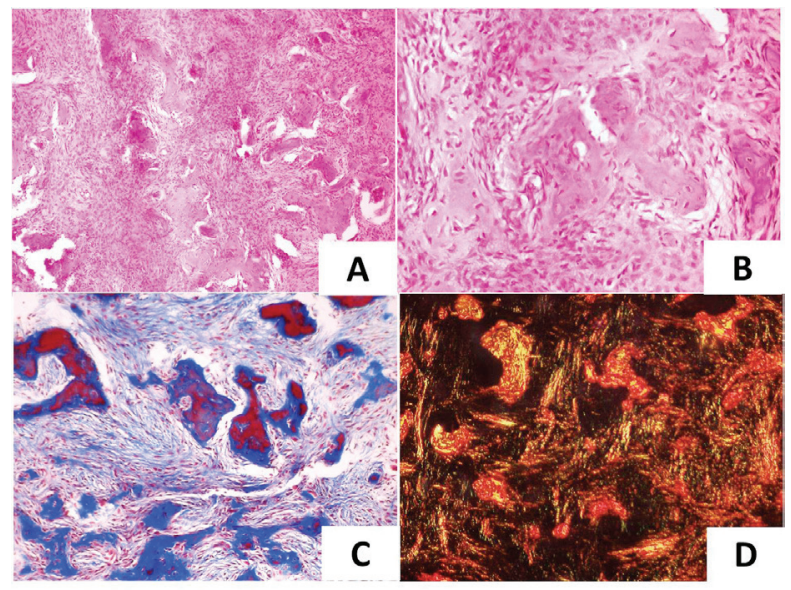

Fonte: Dados da pesquisa

\section{DISCUSSÃO}

O FOC está inserido no grupo de lesões fibro-ósseas benignas dos maxilares (LFOBM), as quais estão representadas por condições clinicopatológicas constituídas por uma mistura de tecido fibrocolagenoso e vários materiais mineralizados, incluindo tecido ósseo imaturo, osso lamelar e cemento (PHATTARATARATIP; PHOLJAROEN; TIRANON, 2013). É considerada uma neoplasia verdadeira, originada de células mesenquimais indiferenciadas presentes no tecido medular (MCCARTHY, 2013).

Os mecanismos fisiopatológicos associados à etiopatogênese do FOC ainda são obscuros, mas estudos têm demonstrado uma possível relação com a inativação do gene HRPT2, um gene supressor de tumor que codifica a proteína inibitória da proliferação celular denominada parafibromina (MESQUITA NETTO ET AL., 2013). Além disso, outro estudo recente também sugeriu uma provável relação entre o desenvolvimento deste tumor e um hiperexpressão do gene RHAMM, responsável pela codificação de receptores para ácido hialurônico associados à motilidade celular (HATANO ET AL., 2013). Contudo, o papel preciso destas alterações genéticas no desenvolvimento e comportamento biológico do FOC não está completamente esclarecido, demandando a realização de novos estudos a fim de proporcionar uma maior elucidação deste tema.

0 perfil epidemiológico do FOC vem sendo bastante estudado ao longo dos anos, ocorrendo especialmente em adultos do sexo feminino e em região posterior de mandíbula (TRIANTAFILLIDOU ET AL., 2012). Assim, o presente caso está em consonância com as características demográficas classicamente descritas para esse tumor. Adicionalmente, a apresentação clínica e padrão de crescimento tumoral observados no corrente caso também se encaixam nas descrições clássicas relatadas na literatura (VIEIRA-QUIEROZ ET AL., 2013).

Os achados imaginológicos observados no presente caso são compatíveis com muitos relatos na literatura (SHEIKHI ET AL., 2013). Contudo, é importante ressaltar que as características radiográficas e/ou tomográficas do FOC dependem fundamentalmente da quantidade e maturação das matrizes cálcicas depositadas; assim, tumores com maior produção de trabéculas ósseas ou ossículos não trabeculares (esferoidais, globulares ou psamomatóides) exibem uma maior densidade imaginológica, expressa por imagens radiopacas (radiografias) ou hiperdensas (tomográficas) mais abundantes (MITHRA; BASKARAN; SATHYAKUMAR ET AL., 2012). Por outro lado, é importante destacar que não existe uma relação entre a intensidade da mineralização e o grau de crescimento tumoral, ou mesmo com o tempo de evolução da lesão nos casos de FOC (ANDRADE ET AL., 2013).

Conquanto exista um grande número de lesões categorizadas como fibro-ósseas, o diagnóstico diferencial imaginológico é de extrema relevância 
para proporcionar dados clínicos estratégicos para o estabelecimento final do diagnóstico (FASOLIS ET AL., 2013). A displasia óssea fibrosa (DOF) representa a principal entidade nosológica a ser distinguida do FOC, mas esta é classicamente observada como uma lesão constituída de uma radiopacidade difusa e irregular (aspecto de "vidro despolido"), com margens bastante imprecisas, frequentemente em região posterior da maxila (FUSCONI ET AL., 2013).

Além disso, o fibroma ossificante juvenil agressivo (FOJA) constitui outra LFOBM cujos achados imaginológicos podem ser confundidos com aqueles evidenciados no FOC. Contudo, a possibilidade de perfuração de corticais ósseas e reabsorção radicular de dentes envolvidos, aliados a um maior acometimento da maxila e em faixas etárias mais baixas representam dados importantes para estabelecimento do diagnóstico diferencial (SLOOTWEG, 2012). Tais relatos conduzem a inferência de que, em casos com este padrão de imagem, a correlação com dados clínicos e demográficos é criteriosa para o estabelecimento de hipóteses de diagnóstico clínico-imaginológico.

Tem sido relatado que o diagnóstico histopatológico do FOC pode constituir um desafio para o patologista, especialmente na ausência de informações clínicas sólidas (PHATTARATARATIP; PHOLJAROEN; TIRANON, 2013). Os autores têm creditado essa dificuldade às similaridades histopatológicas entre o FOC e outras LFOBM, como a DOF e o FOJA (LASISI; ADISA; OLUSANYA, 2014). Os principais achados histológicos que distinguem a DOF do FOC estão representados pela formação de trabeculado ósseo caracteristicamente delgado e curvilíneo (em formato de "C" ou "S"), parca atividade osteoblástica e estroma fibroso maduro, moderadamente celularizado, evidenciados na DOF, em oposição às trabéculas irregulares espessas e com patente pavimentação osteoblástica, e o estroma ricamente celularizado e fasciculado observado no FOC (MOSHY; MWAKYOMA; CHINDIA, 2010).

Por outra ótica, O FOJA exibe um estromaestoriforme e vorticilar hipercelularizado, formação de trabéculas imaturas de osteóide celular (semelhantes àquelas vistas nos osteossarcomas), aglome- rados focais de células gigantes multinucleadas e, ocasionalmente, atividade mitótica elevada (RANGANATH ET AL., 2014), achados não encontrados no FOC. Entretanto, ainda que existam parâmetros relativamente claros para estabelecimento do diagnóstico diferencial histopatológico entre essas entidades, a frequente e considerável superposição entre essas características determina a necessidade de uma criteriosa correlação entre os aspectos clínicos, imaginológicos e histopatológicos como a melhor estratégia de estabelecimento de um diagnóstico seguro para o FOC.

\section{CONCLUSÃO}

No presente trabalho foi apresentado um caso clássico de fibroma ossificante central, com ênfase nos critérios de diagnóstico diferencial. A análise do caso e sua relação com a literatura vigente permitiu concluir que é de extrema relevância que o diagnóstico do FOC seja devidamente embasado numa correlação de achados clinico patológicos para assegurar a instituição do diagnóstico e terapêutica mais adequados.

\section{REFERÊNCIAS}

AGARWAL, N. et al. Recurrent

bimaxillaryradiopacities: A rare case report.

Contemp. Clin. Dent., v.3, n.5, apr., 2012. p.103-8.

ANDRADE, M. et al. Ossifying fibroma of the jaws: a clinicopathological case series study. Braz. Dent. J., v.24, n.6, nov-dez, 2013. p.662-666.

DESAI, K. et al. Bimaxillary presentation of central ossifying fibroma: a unique aggressive entity. BMJ Case Rep., v.16, [on-line], jun., 2013.

FASOLIS, M. et al. Surgical management of synchronous central giant cell granuloma and 
ossifying fibroma of the mandible. J. Craniofac.

Surg., v.24, n.6, nov., 2013. p.627-630.

FUSCONI, M. et al. Fibrous dysplasia of the maxilla: diagnostic reliability of the study image. J. Neurol.

Surg. B. Skull Base., v.74, n.6, dez. 2013. p.364-368.

HATANO, H. et al. Expression of receptor for hyaluronan-mediated motility (RHAMM) in ossifying fibromas. Histol. Histopathol., v.28, n.4, apr. 2013. p.473-480.

HUNASGI, S., RAGHUNATH, V. A clinicopathological study of ossifying fibromas and comparison between central and peripheral ossifying fibromas. J.Contemp.

Dent.Pract., v.13, n.4, jul., 2012. p.509-514.

KONOPKA, W. et al. Ossifying fibroma-cementoma of jaw. Differences in histopathological nomenclature. Otolaryngol. Polska, v.66, 2012. p.359-362

LASISI, T.J., ADISA, A.O., OLUSANYA, A.A. Fibroosseous lesions of the jaws in Ibadan, Nigeria. Oral Health Dent.Manag., v.13, n.1, mar. 2014. p.41-44.

LAUREANO FILHO, J.R. et al. Osteotomialefort I na abordagem cirúrgica Intrabucal para exérese do angiofibroma juvenil Nasofaríngeo: relato de caso clínico. Rev. Cir. Traumatol. Buco Maxilo Fac., v.4, n.4, out-dez. 2004. p.204-257.

MCCARTHY, E.F. Fibro-osseous lesions of the maxillofacial bones. Head Neck Pathol., v.7, n.q, mar. 2013. p.5-10.

MESQUITA NETTO, A.C. et al. Assessing the contribution of HRPT2 to the pathogenesis of jaw fibrous dysplasia, ossifying fibroma, and osteosarcoma. Oral Surg. Oral Med. Oral Pathol. Oral Radiol., v.115, n.3, mar. 2013. p.359-67,

MITHRA, R; BASKARAN, P; SATHYAKUMAR, M.Imaging in the diagnosis of cemento-ossifying fibroma: a case series. J. Clin. Imaging. Sci., v.2, n.52, [on-line], 2012.

MOSHY, J.L.;MWAKYOMA, H.A.;CHINDIA,M.L. Evaluation and histological maturation characteristics of fibrous dysplasia and ossifying fibroma: a case series. East Afr. Med. J., v.87, n.5, maio 2010. p.215-219.

\section{OLIVEIRA, A.C.A. et al. Fibromas Ossificantes}

Centrais e Displasias Fibrosas dos Maxilares:Estudo Clínico, Radiográfico e Histopatológico de 28 Casos.

Pesq. Bras. Odontoped. Clin. Integr., v.8, n.1, janabr. 2008. p.111-115.

PHATTARATARATIP, E.; PHOLJAROEN, C.; TIRANON, P. A Clinicopathologic Analysis of 207 Cases of Benign Fibro-Osseous Lesions of the Jaws. Int. J.Surg.Pathol., v.22, n.4, dec., 2013. p.326-333.

RANGANATH, K. et al. Juvenile Psammomatoid Ossifying Fibroma of Maxillary Sinus: Case Report with Review of Literature. J.Maxillofac. Oral Surg., v.13, n.2, jun. 2014. p.109-114.

RIBEIRO, A.C.P.et al. Bilateral central ossifying fibroma affecting the mandible: report of an uncommon case and critical review of the literature.

Oral Surg. Oral Med. Oral Pathol. Oral Radiol., v.111, n.2, fev. 2011. p.21-26.

SHEIKHI, M. et al. Central cementifying fibroma of maxilla. Dent. Res. J. (Isfahan)., v.10, n.1, jan. 2013. p.122-125.

SILVA, C.O. et al. Localized gingival enlargement associated with alveolar process expansion: peripheral ossifying fibroma coincident with central odontogenic fibroma. J. Periodontol., v.78, n.7, jul. 2007. p.1354-1359.

SLOOTWEG, P.J. Juvenile trabecular ossifying fibroma: an update. VirchowsArch., v.461, n.6, dez. 2012. p.699-703. 
TRIANTAFILLIDOU, K. et al. Ossifying fibroma of the jaws: a clinical study of 14 cases and review of the literature. Oral Surg. Oral Med. Oral Pathol. Oral Radiol., v.114, n.2, ago. 2012. p.193-199.
VIEIRA-QUIEROZ, I. et al. Extensive central ossifying fibroma of the maxilla: a case report. Gen. Dent. v.61, n.3, maio-jun. 2013. p.36-38.

WANG, T.T. et al. Two cases of multiple ossifying fibromas in the jaws. Diagn.Pathol., v.28, n.9, 2014. p.75.

2 Graduando em Odontologia da Universidade Tiradentes -UNIT; Programa Institucional de Bolsas de Iniciação em Desenvolvimento Tecnológico e Inovação - PIBIT/CNPq, Aracaju-SE. Email: lennonrrr@live.com

3 Cirurgiã-dentista pela Universidade Tiradentes - UNIT, Aracaju-SE. Email: candi.rosa@hotmail.com

\begin{abstract}
4 Especialista em Radiologia e Imaginologia Odontológicas pela Faculdade de Odontologia da Universidade Federal da Bahia - UFBA; Mestranda em Odontologia pela Universidade Federal de Sergipe -UFS, Aracaju-SE. Email: ju_melof@hotmail.com

5 Professora Titular do Departamento de Odontologia da Universidade Federal de Sergipe, Aracaju-SE. Email: mbfmelo@infonet.com.br

6 Docente do Programa de Pós-graduação em Saúde e Ambiente da Universidade Tiradentes - UNIT, Aracaju/SE. Email: ricardo.patologia@uol.com.br
\end{abstract}

